\title{
CMV Matrices with Super Exponentially Decaying Verblunsky Coefficients
}

\author{
M. Zinchenko* \\ Department of Mathematics and Statistics, University of New Mexico, Albuquerque, NM 87131, USA

\begin{abstract}
We investigate several equivalent notions of the Jost solution associated with a unitary CMV matrix and provide a necessary and sufficient conditions for the Jost solution to consist of entire functions of finite growth order in terms of super exponential decay of Verblunsky coefficients. We also establish several one-to-one correspondences between CMV matrices with super-exponentially decaying Verblunsky coefficients and spectral data associated with the first component of the Jost solution.
\end{abstract}

Keywords and phrases: Jost solution, CMV matrix, Inverse problem

Mathematics Subject Classification: 47B36, 34L40, 39A70

\section{Introduction}

Five diagonal unitary matrices have been studied extensively in recent years due to their rich connections with orthogonal polynomials on the unit circle as well as function and operator theory. These matrices were introduced first in the context of numerical linear algebra $[4,30]$, but were later rediscovered in the context of orthogonal polynomials on the unit circle [5] and coined CMV matrices in [24]. For a comprehensive discussion of CMV matrices and related topics we refer to the two volume monograph $[26,27]$ and review papers $[24,25,28]$.

In this note we consider infinite CMV matrices, which can be viewed as unitary operators on $\ell^{2}\left(\mathbb{N}_{0}\right)$. The entries of a CMV matrix $U$ are uniquely determined by a sequence of complex numbers in the unit disk $\left\{\alpha_{k}\right\}_{k=1}^{\infty}$ which are called Verblunsky coefficients. For CMV matrices with sufficiently fast decaying Verblunsky coefficients there exists a unique solution $u(z)=\left\{u_{k}(z)\right\}_{k=0}^{\infty}$ of $[(U-z) u(z)]_{k}=0, k \geq 1$, with prescribed asymptotic behavior at infinity. The solution $u(z)$ is called the Jost solution of $U$ and is directly related to the spectral data of the associated CMV matrix $U$. We will study the nonlinear map that takes a sequence of Verblunsky coefficients to $u_{0}(z)$, the first component of the Jost solution of the corresponding CMV matrix $U$. This map is an analog of the spectral transformation that provides a one-to-one correspondence between sequences of Verblunsky coefficients and probability measures on the unit circle representing the spectral measures of the corresponding CMV matrices.

In spectral theory, one is interested in finding relations between coefficients of an operator and properties of its spectral data. There is a long history of such results in the context of orthogonal polynomials

${ }^{*}$ Corresponding author. E-mail: maxim@math.unm.edu 
on the unit circle $[26,27]$. We mention a few of the most remarkable ones below. The spectral theorem for CMV matrices combined with the Szegö theorem [26, Thm.2.3.1] shows that there is a one-to-one correspondence between $\ell^{2}$ Verblunsky coefficients and probability measures $d \mu=w \frac{d \theta}{2 \pi}+d \mu_{s}$ on the unit circle with integrable $\log w$. Likewise, the spectral theorem combined with the Baxter theorem [26, Thm.5.2.1] gives a one-to-one correspondence between $\ell^{1}$ Verblunsky coefficients and probability measures $d \mu=w \frac{d \theta}{2 \pi}+d \mu_{s}$ on the unit circle with $\ell^{1}$ Fourier coefficients and $w$ being uniformly bounded away from zero. Similarly, the Nevai-Totik theorem [26, Thm.7.1.3] provides necessary and sufficient conditions for exponential decay of Verblunsky coefficients in terms of analytic properties of the corresponding Szegö function.

The main goal of this paper is to investigate the case of Verblunsky coefficients with super exponential decay of the form $\left|\alpha_{k}\right| \leq c k^{-k / \delta}$ and find the corresponding conditions on the spectral data. Motivated by the approach used in [6] for the study of Jacobi matrices with exponentially decaying coefficients, we establish necessary and sufficient conditions for the existence of the Jost solution $\left\{u_{k}(z)\right\}_{k=0}^{\infty}$ with entire functions $u_{k}(z), k \geq 0$, of finite growth order in terms of super exponential decay of Verblunsky coefficients. We also obtain one-to-one correspondences between the class of super exponentially decaying Verblunsky coefficients, the class of entire functions of finite growth order vanishing at the origin that represent $u_{0}(z)$, and the class of possible sets of zeros of $u_{0}(z)$ which we describe explicitly.

The paper is organized as follows. In Section 2 we introduce the basics of CMV matrices and state some known facts. Section 3 is devoted to a discussion of three alternative notions of the Jost solution which we show to be equivalent. Finally, in Sections 4 and 5 we prove our main results.

We use the following notation in the paper. The set of nonnegative integers is denoted by $\mathbb{N}_{0}$ and the Hilbert space of all square summable complex-valued sequences by $\ell^{2}\left(\mathbb{N}_{0}\right)$. The inner product in the Hilbert space $\ell^{2}\left(\mathbb{N}_{0}\right)$ is denoted by $\langle\cdot, \cdot\rangle$ and is assumed to be linear in the second argument. The vectors of the canonical basis of $\ell^{2}\left(\mathbb{N}_{0}\right)$ are denoted by $\delta_{k}, k \geq 0$. The open unit disk in the complex plane is denoted by $\mathbb{D}$.

\section{Preliminaries}

Below we summarize some of the results established in [9] that will be used throughout the paper. A CMV matrix $U$ with Verblunsky coefficients $\left\{\alpha_{k}\right\}_{k=1}^{\infty} \subset \mathbb{D}$ is defined by

$$
U=\left(\begin{array}{ccccccc}
-\alpha_{1} & \rho_{1} & 0 & & & & \\
-\rho_{1} \alpha_{2} & -\bar{\alpha}_{1} \alpha_{2} & -\rho_{2} \alpha_{3} & \rho_{2} \rho_{3} & & 0 \\
\rho_{1} \rho_{2} & \bar{\alpha}_{1} \rho_{2} & -\bar{\alpha}_{2} \alpha_{3} & \bar{\alpha}_{2} \rho_{3} & 0 & & \\
& 0 & -\rho_{3} \alpha_{4} & -\bar{\alpha}_{3} \alpha_{4} & -\rho_{4} \alpha_{5} & \rho_{4} \rho_{5} & \\
& & \rho_{3} \rho_{4} & \bar{\alpha}_{3} \rho_{4} & -\bar{\alpha}_{4} \alpha_{5} & \bar{\alpha}_{4} \rho_{5} & 0 \\
0 & & \ddots & \ddots & \ddots & \ddots & \ddots
\end{array}\right)
$$

where $\rho_{k}=\sqrt{1-\left|\alpha_{k}\right|^{2}}, k \geq 1$. The CMV matrix $U$ admits a factorization into a product of two block diagonal matrices $U=V W$. The matrices $V$ and $W$ are given by

$$
V=1 \oplus\left(\bigoplus_{k=1}^{\infty} \Theta_{2 k}\right), \quad W=\bigoplus_{k=1}^{\infty} \Theta_{2 k-1},
$$

where 1 denotes a $1 \times 1$ block and $\Theta_{k}$ denote $2 \times 2$ blocks

$$
\Theta_{k}=\left(\begin{array}{cc}
-\alpha_{k} & \rho_{k} \\
\rho_{k} & \bar{\alpha}_{k}
\end{array}\right), \quad k \geq 1
$$

It is easy to see that the matrices $V, W$, and hence, $U$ define unitary operators on $\ell^{2}\left(\mathbb{N}_{0}\right)$. 
The CMV recurrence equation is defined for all $z \in \mathbb{C} \backslash\{0\}$ by

$$
\left(\begin{array}{c}
u_{k}(z) \\
v_{k}(z)
\end{array}\right)=T_{k}(z)\left(\begin{array}{c}
u_{k-1}(z) \\
v_{k-1}(z)
\end{array}\right), \quad k \geq 1
$$

where,

$$
T_{k}(z)=\left\{\begin{array}{cl}
\frac{1}{\rho_{k}}\left(\begin{array}{cc}
\alpha_{k} & z \\
1 / z & \bar{\alpha}_{k}
\end{array}\right), & k \text { odd } \\
\frac{1}{\rho_{k}}\left(\begin{array}{cc}
\bar{\alpha}_{k} & 1 \\
1 & \alpha_{k}
\end{array}\right), & k \text { even }
\end{array}\right.
$$

It is related to the CMV matrix via the following result obtained in [9].

Lemma 2.1. For every $z \in \mathbb{C} \backslash\{0\}$, the sequence $\left(\begin{array}{l}u(z) \\ v(z)\end{array}\right)=\left\{\left(\begin{array}{l}u_{k}(z) \\ v_{k}(z)\end{array}\right)\right\}_{k=0}^{\infty}$ is a solution of the CMV recursion equation (2.4) if and only if

$$
\left\{\begin{array}{l}
V v(z)=u(z)+\left[v_{0}(z)-u_{0}(z)\right] \delta_{0} \\
U u(z)=z u(z)+z\left[v_{0}(z)-u_{0}(z)\right] \delta_{0}
\end{array}\right.
$$

The Weyl-Titchmarsh $m$-function of a CMV matrix $U$ is defined by

$$
m_{0}(z)=\left\langle\delta_{0},(U+z)(U-z)^{-1} \delta_{0}\right\rangle=1+2\left\langle\delta_{0}, z(U-z)^{-1} \delta_{0}\right\rangle, \quad z \in \mathbb{D} .
$$

The spectral theorem then gives

$$
m_{0}(z)=\int_{-\pi}^{\pi} \frac{\mathrm{e}^{i t}+z}{\mathrm{e}^{i t}-z} d \mu_{0}(t), \quad z \in \mathbb{D},
$$

where $d \mu_{0}$ denotes the spectral measure associated with the unitary operator $U$ and the cyclic vector $\delta_{0}$. Since $\left(\mathrm{e}^{i t}+z\right) /\left(\mathrm{e}^{i t}-z\right)$ has positive real part for all $z \in \mathbb{D}$, it follows that $m_{0}(z)$ is a Carathéodory function (i.e., analytic with $\operatorname{Re} m_{0}(z)>0$ on $\mathbb{D}$ ). Another important function associated with $U$ is the Schur function $f_{0}(z)$ (i.e., analytic with $\left|f_{0}(z)\right|<1$ on $\mathbb{D}$ ) defined by

$$
f_{0}(z)=\frac{1}{z} \frac{m_{0}(z)-1}{m_{0}(z)+1}, \quad z \in \mathbb{D} .
$$

Employing the Neumann series for $(U-z)^{-1}$ in (2.6) and using (2.8) yields the asymptotics

$$
m_{0}(z)=1+2 \sum_{n=1}^{\infty} z^{n}\left\langle\delta_{0}, U^{-n} \delta_{0}\right\rangle=1-2 \bar{\alpha}_{1} z+O\left(z^{2}\right) \text { and } f_{0}(0)=-\bar{\alpha}_{1} .
$$

There is a one-to-one correspondence provided by the spectral theorem for CMV matrices between the class of Verblunsky coefficients (equiv., CMV matrices) and the class of Schur functions (equiv., Carathéodory function normalized by $\left.m_{0}(0)=1\right)$.

\section{The Jost solution of a CMV matrix}

In this section, we introduce three equivalent notions of the Jost solution associated with a CMV matrix and explore some of their properties and connections with the Weyl-Titchmarsh $m$-function $m_{0}(z)$ and the Schur functions $f_{n}(z)$ associated with the truncated Verblunsky coefficients $\left\{\alpha_{n+k}\right\}_{k=1}^{\infty}, n \geq 0$.

First, consider the free CMV matrix which is the CMV matrix $U$ with the identically zero Verblunsky coefficients $\alpha_{k}=0, k \geq 1$. In this case the Jost solution $u(z)=\left\{u_{k}(z)\right\}_{k=0}^{\infty}$ is defined to be the solution 
of $(U-z) u(z)=z \delta_{0}$. It is easy to check that the Jost solution of the free CMV matrix is given by $u_{2 k-1}(z)=z^{k}, u_{2 k}(z)=0, k \geq 1, z \in \mathbb{C}$.

Now let $\Omega$ be an open domain on $\mathbb{C}$ containing the origin and consider a general CMV matrix $U$. A sequence of analytic functions $u(z)=\left\{u_{k}(z)\right\}_{k=0}^{\infty}$ on $\Omega$ is called the Jost solution of $U$ on $\Omega$ if

$$
(U-z) u(z)=z \psi_{0}(z) \delta_{0}, \quad z \in \Omega,
$$

for some function $\psi_{0}(z)$, called the Jost function of $U$ on $\Omega$, and

$$
\lim _{k \rightarrow \infty} \frac{u_{2 k-1}(z)}{z^{k}}=1, \quad \lim _{k \rightarrow \infty} \frac{u_{2 k}(z)}{z^{k}}=0, \quad z \in \Omega .
$$

We will show shortly that a necessary condition for the existence of the Jost solution is the decay of Verblunsky coefficients $\alpha_{k} \rightarrow 0$ as $k \rightarrow \infty$.

Next, we introduce the Jost solution of the CMV recursion equation as an analytic solution $\left(\begin{array}{l}u(z) \\ v(z)\end{array}\right)=$ $\left\{\left(\begin{array}{c}u_{k}(z) \\ v_{k}(z)\end{array}\right)\right\}_{k=0}^{\infty}$ of (2.4) on $\Omega$ such that $u(z)$ satisfies (3.2) and $v(z)$ satisfies

$$
\lim _{k \rightarrow \infty} \frac{v_{2 k-1}(z)}{z^{k}}=0, \quad \lim _{k \rightarrow \infty} \frac{v_{2 k}(z)}{z^{k}}=1, \quad z \in \Omega .
$$

The above two notions of the Jost solution are, in fact, equivalent. Indeed, if $\left(\begin{array}{l}u(z) \\ v(z)\end{array}\right)$ is the Jost solution of the CMV recursion equation (2.4), then by Lemma $2.1 u(z)$ is the Jost solution of $U$ and the Jost function of $U$ is given by

$$
\psi_{0}(z)=v_{0}(z)-u_{0}(z)
$$

The converse is also true. If $u(z)$ is the Jost solution of $U$ on $\Omega$, define the complementary sequence $v(z)$ by $v_{k}(z)=\left[V^{-1} u(z)\right]_{k}, k \geq 1$, and $v_{0}(z)=z^{-1}[W u(z)]_{0}$. Then $\left(\begin{array}{l}u(z) \\ v(z)\end{array}\right)$ satisfies (2.4) by Lemma 2.1. Moreover, the structure of the matrix $V$ in (2.2) and the $2 \times 2$ blocks $\Theta_{k}$ in (2.3) shows that

$$
\left(\begin{array}{c}
v_{2 k-1}(z) \\
v_{2 k}(z)
\end{array}\right)=\left(\begin{array}{cc}
-\bar{\alpha}_{2 k} & \rho_{2 k} \\
\rho_{2 k} & \alpha_{2 k}
\end{array}\right)\left(\begin{array}{c}
u_{2 k-1}(z) \\
u_{2 k}(z)
\end{array}\right), \quad k \geq 1
$$

It follows that $\left\{v_{k}(z)\right\}_{k=1}^{\infty}$ are analytic on $\Omega$ since $\left\{u_{k}(z)\right\}_{k=1}^{\infty}$ are. In addition, by (3.5), the asymptotic (3.3) follows from (3.2) as long as $\alpha_{k} \rightarrow 0$ as $k \rightarrow \infty$. Thus, it remains to verify that $\alpha_{k} \rightarrow 0$ as $k \rightarrow \infty$ and show that $v_{0}(z)$ does not have a pole at $z=0$. In fact, we will show that all the quotients in (3.2) and (3.3) do not have poles at the origin, and hence, are analytic on $\Omega$.

In the following it will be convenient to change variables to

$$
\left(\begin{array}{l}
x_{k}(z) \\
y_{k}(z)
\end{array}\right)=\left(\begin{array}{cc}
z^{\lfloor k / 2\rfloor} & 0 \\
0 & z^{\lceil k / 2\rceil}
\end{array}\right)^{-1}\left(\begin{array}{ll}
0 & 1 \\
1 & 0
\end{array}\right)^{k}\left(\begin{array}{l}
u_{k}(z) \\
v_{k}(z)
\end{array}\right), \quad k \geq 0, z \in \Omega \backslash\{0\},
$$

and extend (3.6) by analyticity to $z=0$. A priori the functions $x_{k}(z), y_{k}(z)$ are analytic on $\Omega \backslash\{0\}$ but might have a pole at $z=0$. To simplify notation we will consider $x_{k}(z), y_{k}(z)$ as functions to the extended complex plane, that is, we will define them on all of $\Omega$ by assigning the value infinity at the pole. The CMV recursion equation (2.4) in the new variables becomes

$$
\left(\begin{array}{l}
x_{k}(z) \\
y_{k}(z)
\end{array}\right)=\frac{1}{\rho_{k+1}}\left(\begin{array}{cc}
z & -\bar{\alpha}_{k+1} z \\
-\alpha_{k+1} & 1
\end{array}\right)\left(\begin{array}{l}
x_{k+1}(z) \\
y_{k+1}(z)
\end{array}\right), \quad k \geq 0, z \in \Omega .
$$

It is easy to see that (3.2) is equivalent to $x_{2 k}(z) \rightarrow 0$ and $y_{2 k-1}(z) \rightarrow 1$ as $k \rightarrow \infty$, hence, $x_{2 k}(0)$ and $y_{2 k-1}(0)$ must be finite for all sufficiently large $k$. Then it follows from (3.7) that $x_{k}(0)$ and $y_{k}(0)$ are finite for all $k \geq 0$, and hence, $v_{0}(0)$ as well as all the quotients in (3.2) and (3.3) are finite at $z=0$ 
by (3.6). In addition, (3.7) show that $x_{k}(0)=0$ and $y_{k}(0) \rho_{k+1}=y_{k+1}(0)$ for all $k \geq 0$. In particular, $y_{k}(0)=y_{0}(0) \prod_{n=1}^{k} \rho_{n}, k \geq 0$, and hence, $y_{k}(0)$ is monotone decreasing w.r.t. $k$. The asymptotic $y_{2 k-1}(0) \rightarrow 1$ as $k \rightarrow \infty$ then implies

$$
1 \leq y_{0}(0)=\prod_{n=1}^{\infty} \frac{1}{\rho_{n}}<\infty .
$$

Thus, $\rho_{k} \rightarrow 1$, and hence, $\alpha_{k} \rightarrow 0$ as $k \rightarrow \infty$. This finishes the verification of the equivalence of the two notions of the Jost solution.

It follows from the above discussion that in the new variables we have the third equivalent notion of the Jost solution which is an analytic solution $\left(\begin{array}{l}x(z) \\ y(z)\end{array}\right)$ of (3.7) on $\Omega$ with the asymptotic

$$
\lim _{k \rightarrow \infty}\left(\begin{array}{l}
x_{k}(z) \\
y_{k}(z)
\end{array}\right)=\left(\begin{array}{l}
0 \\
1
\end{array}\right), \quad z \in \Omega .
$$

The Jost solution $\left(\begin{array}{l}x(z) \\ y(z)\end{array}\right)$ of (3.7) on $\Omega=\mathbb{C}$ has a useful symmetry property which we explore next. Define

$$
x_{k}^{\#}(z)=\overline{x_{k}(1 / \bar{z})}, \quad y_{k}^{\#}(z)=\overline{y_{k}(1 / \bar{z})}, \quad k \geq 0, z \in \mathbb{C} \backslash\{0\},
$$

and note that the matrix in (3.7) has a symmetry that allows us to rewrite (3.7) as

$$
\left(\begin{array}{l}
y_{k}^{\#}(z) \\
x_{k}^{\#}(z)
\end{array}\right)=\frac{1}{\rho_{k+1}}\left(\begin{array}{cc}
z & -\bar{\alpha}_{k+1} z \\
-\alpha_{k+1} & 1
\end{array}\right)\left(\begin{array}{l}
\frac{1}{z} y_{k+1}^{\#}(z) \\
\frac{1}{z} x_{k+1}^{\#}(z)
\end{array}\right), \quad k \geq 0, z \in \mathbb{C} \backslash\{0\} .
$$

Combining (3.11) and (3.7) into a single matrix equation and taking the $2 \times 2$ determinants on both sides then gives

$$
y_{k}^{\#}(z) y_{k}(z)-x_{k}^{\#}(z) x_{k}(z)=y_{k+1}^{\#}(z) y_{k+1}(z)-x_{k+1}^{\#}(z) x_{k+1}(z),
$$

that is, the expression on the LHS is constant w.r.t. $k$. Utilizing the asymptotic (3.9) then yields the following useful identity

$$
y_{k}^{\#}(z) y_{k}(z)-x_{k}^{\#}(z) x_{k}(z)=1, \quad k \geq 0, z \in \mathbb{C} \backslash\{0\} .
$$

Next, we derive several relations between the Jost solution, Jost function, $m$-function, and Schur function. As before, let $\left(\begin{array}{l}x(z) \\ y(z)\end{array}\right)$ be the Jost solution of (3.7) on $\Omega \supset \mathbb{D}$. Since, by (3.6), $u_{0}(z)=x_{0}(z)$ and $v_{0}(z)=y_{0}(z),(3.4)$ yields

$$
\psi_{0}(z)=y_{0}(z)-x_{0}(z), \quad z \in \Omega \text {. }
$$

Combining (2.6) and (3.1) gives

$$
m_{0}(z)=1+2 \frac{u_{0}(z)}{\psi_{0}(z)}=\frac{y_{0}(z)+x_{0}(z)}{y_{0}(z)-x_{0}(z)}, \quad z \in \mathbb{D}
$$

and hence by $(2.8)$,

$$
f_{0}(z)=\frac{1}{z} \frac{x_{0}(z)}{y_{0}(z)}, \quad z \in \mathbb{D} .
$$

Since $f_{0}(z)$ is analytic on $\mathbb{D}$ and, by $(3.13), x_{0}(z)$ and $y_{0}(z)$ have no common zeros on $\mathbb{C} \backslash\{0\}$, it follows from (3.8) and (3.16) that

$$
y_{0}(z) \neq 0, \quad z \in \mathbb{D}
$$


Consider the CMV matrix $U_{n}$ associated with the truncated sequence of Verblunsky coefficients $\left\{\alpha_{n+k}\right\}_{k=1}^{\infty}$ and denote by $f_{n}(z)$ the Schur function associated with $U_{n}$. Then noting that the Jost solution of (3.7) associated with $U_{n}$ is the truncation of the Jost solution of (3.7) associated with $U$ gives

$$
f_{n}(z)=\frac{1}{z} \frac{x_{n}(z)}{y_{n}(z)}, \quad n \geq 0, z \in \mathbb{D} .
$$

In addition, similar to $(2.9)$, we obtain

$$
f_{n}(0)=-\bar{\alpha}_{n+1}, \quad n \geq 0 .
$$

Finally, we note that (3.16) shows that the Jost solution $\left(\begin{array}{l}x(z) \\ y(z)\end{array}\right)$, and hence also all the other equivalent forms of the Jost solution and the Jost function, are unique. Indeed, $x_{0}(z)$ and $y_{0}(z)$ are uniquely determined up to a common multiple by $U$ via the Schur function $f_{0}(z)$ and the relation (3.16). Then, by (3.7), the whole Jost solution is determined by $U$ up to the same common multiple and the asymptotic (3.9) shows that the common multiple must be identically 1.

\section{Characterization of CMV matrices with super exponentially decaying Verblunsky coefficients}

In this section we present our first main result. It characterizes CMV matrices that have the Jost solutions on $\mathbb{C}$ whose components are entire functions of finite growth order in terms of super exponential decay of Verblunsky coefficients. This result extends the sufficient condition for the existence of the entire Jost solutions of growth order zero obtained in [31]. Our extension is threefold: we replace the earlier sufficient condition of [31] by a weaker condition, show that the weaker sufficient condition is also necessary, and discuss the Jost solutions of all finite growth orders.

Theorem 4.1. Let $U$ be a CMV matrix with Verblunsky coefficients $\left\{\alpha_{k}\right\}_{k=1}^{\infty} \subset \mathbb{D}$. Then $U$ has the Jost solution $u(z)=\left\{u_{k}(z)\right\}_{k=0}^{\infty}$ on $\mathbb{C}$ with $u_{0}(z)$ being an entire function of growth order at most $\gamma$, that is,

$$
\limsup _{R \rightarrow \infty} \frac{\log \log \max _{|z|=R}\left|u_{0}(z)\right|}{\log R} \leq \gamma
$$

if and only if the Verblunsky coefficients satisfy the super exponential decay condition

$$
\limsup _{k \rightarrow \infty} \frac{k \log (k)}{-\log \left|\alpha_{k}\right|} \leq \gamma
$$

Remark 4.2. (i) (4.1) is equivalent to $\left|u_{0}(z)\right| \leq c(\delta) \exp \left(|z|^{\delta}\right), z \in \mathbb{C}$, for all $\delta>\gamma$.

(ii) (4.2) is equivalent to $\left|\alpha_{k}\right| \leq c(\delta) k^{-k / \delta}, k \geq 1$, for all $\delta>\gamma$.

(iii) Due to the equivalence of (4.1) and (4.2), the inequalities in (4.1) and (4.2) can be replaced by the equalities.

(iv) It follows from the proof that (4.2) implies (4.1) for all $u_{k}(z)$.

Proof. We start by showing that (4.2) implies (4.1). Adopting the approach used in [31], we introduce the constants

$$
C_{k}=\prod_{j=k+1}^{\infty} \frac{1}{\rho_{j}}, \quad k \geq 0
$$

and consider the following Volterra sum equation

$$
\left(\begin{array}{l}
x_{k}(z) \\
y_{k}(z)
\end{array}\right)=C_{k}\left(\begin{array}{l}
0 \\
1
\end{array}\right)-\sum_{n=k+1}^{\infty} \frac{C_{k}}{C_{n}}\left(\begin{array}{cc}
0 & z^{n-k} \bar{\alpha}_{n} \\
\alpha_{n} & 0
\end{array}\right)\left(\begin{array}{l}
x_{n}(z) \\
y_{n}(z)
\end{array}\right), \quad k \geq 0, z \in \mathbb{C} .
$$


It is a straightforward verification that a solution of (4.4) satisfies (3.7), (3.9) and hence gives rise to the Jost solution of $U$ via (3.6). Indeed, if $\left(\begin{array}{l}x_{k}(z) \\ y_{k}(z)\end{array}\right)$ is a solution of (4.4), then the asymptotic (3.9) with $\Omega=\mathbb{C}$ follows from the convergence of the product in (4.3) and the series in (4.4). The recurrence relation (3.7) can be derived from (4.4) as follows,

$$
\begin{aligned}
\left(\begin{array}{l}
x_{k}(z) \\
y_{k}(z)
\end{array}\right)= & C_{k}\left(\begin{array}{l}
0 \\
1
\end{array}\right)-\sum_{n=k+1}^{\infty} \frac{C_{k}}{C_{n}}\left(\begin{array}{cc}
0 & z^{n-k} \bar{\alpha}_{n} \\
\alpha_{n} & 0
\end{array}\right)\left(\begin{array}{l}
x_{n}(z) \\
y_{n}(z)
\end{array}\right) \\
= & C_{k+1} \frac{1}{\rho_{k+1}}\left(\begin{array}{ll}
z & 0 \\
0 & 1
\end{array}\right)\left(\begin{array}{l}
0 \\
1
\end{array}\right)-\frac{1}{\rho_{k+1}}\left(\begin{array}{cc}
0 & z \bar{\alpha}_{k+1} \\
\alpha_{k+1} & 0
\end{array}\right)\left(\begin{array}{l}
x_{k+1}(z) \\
y_{k+1}(z)
\end{array}\right) \\
& -\frac{1}{\rho_{k+1}}\left(\begin{array}{ll}
z & 0 \\
0 & 1
\end{array}\right) \sum_{n=k+2}^{\infty} \frac{C_{k+1}}{C_{n}}\left(\begin{array}{ccc}
0 & z^{n-k-1} \bar{\alpha}_{n} \\
\alpha_{n} & 0
\end{array}\right)\left(\begin{array}{l}
x_{n}(z) \\
y_{n}(z)
\end{array}\right) \\
= & \frac{1}{\rho_{k+1}}\left(\begin{array}{ll}
z & 0 \\
0 & 1
\end{array}\right)\left(\begin{array}{c}
x_{k+1}(z) \\
y_{k+1}(z)
\end{array}\right)-\frac{1}{\rho_{k+1}}\left(\begin{array}{cc}
0 & z \bar{\alpha}_{k+1} \\
\alpha_{k+1} & 0
\end{array}\right)\left(\begin{array}{l}
x_{k+1}(z) \\
y_{k+1}(z)
\end{array}\right) \\
= & \frac{1}{\rho_{k+1}}\left(\begin{array}{cc}
z & -z \bar{\alpha}_{k+1} \\
-\alpha_{k+1} & 1
\end{array}\right)\left(\begin{array}{l}
x_{k+1}(z) \\
y_{k+1}(z)
\end{array}\right), \quad k \geq 0, z \in \mathbb{C} .
\end{aligned}
$$

Thus, it suffices to show that (4.4) has an analytic solution of growth order at most $\gamma$.

We build a solution of (4.4) using the standard Volterra iterations

$$
\left(\begin{array}{l}
x_{k}(z) \\
y_{k}(z)
\end{array}\right)=\sum_{s=0}^{\infty}\left(\begin{array}{l}
x_{k}^{(s)}(z) \\
y_{k}^{(s)}(z)
\end{array}\right)
$$

where

$$
\left(\begin{array}{l}
x_{k}^{(0)}(z) \\
y_{k}^{(0)}(z)
\end{array}\right)=C_{k}\left(\begin{array}{l}
0 \\
1
\end{array}\right), \quad\left(\begin{array}{l}
x_{k}^{(s+1)}(z) \\
y_{k}^{(s+1)}(z)
\end{array}\right)=-\sum_{n=k+1}^{\infty} \frac{C_{k}}{C_{n}}\left(\begin{array}{cc}
0 & z^{n-k} \bar{\alpha}_{n} \\
\alpha_{n} & 0
\end{array}\right)\left(\begin{array}{l}
x_{n}^{(s)}(z) \\
y_{n}^{(s)}(z)
\end{array}\right), \quad k, s \geq 0 .
$$

Then recursively estimating the terms as in [29, Lemma 7.8] using the $\mathbb{C}^{2}$-norm we obtain

$$
\left\|\left(\begin{array}{c}
x_{k}^{(s)}(z) \\
y_{k}^{(s)}(z)
\end{array}\right)\right\| \leq \frac{1}{s !}\left(\left(\prod_{j=1}^{\infty} \frac{1}{\rho_{j}}\right) \sum_{n=k+1}^{\infty}\left|\alpha_{n}\right| \max \left\{1,|z|^{n}\right\}\right)^{s}\left\|\left(\begin{array}{c}
x_{k}^{(0)}(z) \\
y_{k}^{(0)}(z)
\end{array}\right)\right\|, \quad k, s \geq 0
$$

and hence,

$$
\left\|\left(\begin{array}{l}
x_{k}(z) \\
y_{k}(z)
\end{array}\right)\right\| \leq \exp \left(\left(\prod_{j=1}^{\infty} \frac{1}{\rho_{j}}\right) \sum_{n=k+1}^{\infty}\left|\alpha_{n}\right| \max \left\{1,|z|^{n}\right\}\right)\left\|\left(\begin{array}{c}
x_{k}^{(0)}(z) \\
y_{k}^{(0)}(z)
\end{array}\right)\right\|, \quad k \geq 0 .
$$

The assumption of the super exponential decay of Verblunsky coefficients (4.2) guarantees that the constant in the estimate (4.8) is uniformly bounded w.r.t. $z$ on compact subsets of $\mathbb{C}$, hence the series (4.6) converges locally uniformly. Since by construction (4.7) the summands of (4.6) are entire then so is the solution $\left(\begin{array}{l}x_{k}(z) \\ y_{k}(z)\end{array}\right)$ of $(4.4)$.

Next, we estimate the growth order of the entire functions $x_{k}(z)$ and $y_{k}(z)$. It follows from (4.2) that for any $\delta>\gamma$ there exists $c=c(\delta)>0$ such that $\left|\alpha_{n}\right| \leq c n^{-n / \delta}, n \geq 1$. Let $|z|=R>1$ and $N=\left\lfloor(2 R)^{\delta}\right\rfloor$. Then $\left|\alpha_{n} z^{n}\right| \leq c 2^{-n}$ for all $n \geq N+1$, and hence, it follows from (4.9) that

$$
\left\|\left(\begin{array}{l}
x_{N}(z) \\
y_{N}(z)
\end{array}\right)\right\| \leq\left(\prod_{j=1}^{\infty} \frac{1}{\rho_{j}}\right) \exp \left(c \prod_{j=1}^{\infty} \frac{1}{\rho_{j}}\right)
$$


Taking $R$ sufficiently large so that $N \geq k$, applying the recursion (3.7), and estimating the norm of the matrix in (3.7) by $\left\|\left(\begin{array}{cc}z & -\bar{\alpha}_{n} z \\ -\alpha_{n} & 1\end{array}\right)\right\| \leq R\left(1+\left|\alpha_{n}\right|\right)$, then yields

$$
\left\|\left(\begin{array}{l}
x_{k}(z) \\
y_{k}(z)
\end{array}\right)\right\| \leq R^{N-k}\left(\prod_{n=k+1}^{N} \rho_{n}^{-1}\left(1+\left|\alpha_{n}\right|\right)\right)\left\|\left(\begin{array}{c}
x_{N}(z) \\
y_{N}(z)
\end{array}\right)\right\| \leq C R^{(2 R)^{\delta}},
$$

where the constant $C$ is $z$-independent. Thus, we compute

$$
\lim _{R \rightarrow \infty} \frac{\log \log \max _{|z|=R}\left\|\left(\begin{array}{c}
x_{k}(z) \\
y_{k}(z)
\end{array}\right)\right\|}{\log (R)} \leq \delta .
$$

Since $\delta>\gamma$ was arbitrary, it follows that the growth order of $x_{k}(z), y_{k}(z), k \geq 0$, is at most $\gamma$, and hence, (4.1) follows from (3.6).

For the other direction, (4.1) implies (4.2), we start by recalling the equivalent notion of the Jost solution $\left(\begin{array}{l}x(z) \\ y(z)\end{array}\right)$ of the recurrence equation (3.7). As discussed in Section 2, the functions $x_{k}(z), y_{k}(z)$, $k \geq 0$, are entire and $x_{0}(z)$ is of growth order at most $\gamma$ by (4.1). We also recall the definitions of $x_{k}^{\#}(z)$ and $y_{k}^{\#}(z)$ in $(3.10)$, define $\left.f_{k}^{\#}(z)=\overline{f_{k}(1 / \bar{z}}\right)$, note that $z^{-1} f_{k}^{\#}(z)=x_{k}^{\#}(z) / y_{k}^{\#}(z)$ by $(3.18)$, and rewrite (3.13) as,

$$
y_{k}(z)=y_{k}^{\#}(z)^{-1}+z^{-1} f_{k}^{\#}(z) x_{k}(z), \quad k \geq 0, z \in \mathbb{C} \backslash\{0\} .
$$

In the following we restrict all functions to the circle $z=R e^{i \theta}, R>1$, and consider them as elements of $L^{2}\left([-\pi, \pi] ; \frac{d \theta}{2 \pi}\right)$. Let $P_{+}$denote the orthogonal projection in $L^{2}\left([-\pi, \pi] ; \frac{d \theta}{2 \pi}\right)$ onto the span of $\left\{e^{i n \theta}\right\}_{n=1}^{\infty}$. Then applying $P_{+}$to both sides of (4.13) and evaluating the $L^{2}$-norms then yields

$$
\left\|y_{k}-y_{k}(0)\right\|=\left\|P_{+}\left(z^{-1} f_{k}^{\#} x_{k}\right)\right\| \leq\left\|z^{-1} f_{k}^{\#} x_{k}\right\| \leq \frac{1}{R}\left\|f_{k}^{\#} x_{k}\right\| \leq \frac{1}{R}\left\|x_{k}\right\| .
$$

Similarly, rewriting the first component of (3.7) as

$$
z^{-1} \rho_{k} x_{k-1}(z)=x_{k}(z)-\bar{\alpha}_{k} y_{k}(z), \quad k \geq 1,
$$

applying $P_{+}$to both sides, and estimating the $L^{2}$-norms gives

$$
\begin{aligned}
\frac{1}{R}\left\|x_{k-1}\right\| & \geq\left\|z^{-1} \rho_{k} x_{k-1}\right\| \geq\left\|P_{+}\left(z^{-1} \rho_{k} x_{k-1}\right)\right\|=\left\|P_{+}\left(x_{k}-\bar{\alpha}_{k} y_{k}\right)\right\| \\
& \geq\left\|P_{+} x_{k}\right\|-\left|\alpha_{k}\right|\left\|P_{+} y_{k}\right\| \geq\left\|x_{k}-x_{k}(0)\right\|-\left\|y_{k}-y_{k}(0)\right\| .
\end{aligned}
$$

Recalling that $x_{k}(0)=0$ and combining (4.16) with (4.14) then implies

$$
\left\|x_{k-1}\right\| \geq(R-1)\left\|x_{k}\right\|, \quad k \geq 1 .
$$

Let $\tilde{x}_{k}(z)=z^{-1} x_{k}(z)$ and recall that $-\bar{\alpha}_{k+1}=\tilde{x}_{k}(0) / y_{k}(0)$ by (3.18) and (3.19). Thus, using the mean value property of the entire function $\tilde{x}_{k}(z)$ and iterating the inequality (4.17), we obtain

$$
\left|\alpha_{k+1}\right|=\frac{\left|\tilde{x}_{k}(0)\right|}{\left|y_{k}(0)\right|} \leq \frac{\left\|\tilde{x}_{k}\right\|}{\left|y_{k}(0)\right|} \leq \frac{\left\|x_{k}\right\|}{\left|y_{k}(0)\right|} \leq \frac{1}{R-1} \frac{\left\|x_{k-1}\right\|}{\left|y_{k}(0)\right|} \leq \frac{1}{(R-1)^{k}} \frac{\left\|x_{0}\right\|}{\left|y_{k}(0)\right|} .
$$

Since $x_{0}(z)$ is of growth order at most $\gamma$, for any $\delta>\gamma$ there exists $c=c(\delta)>0$ such that $\left|x_{0}(z)\right| \leq$ $c \exp \left(|z|^{\delta}\right)$. Hence, we obtain from (4.18) that

$$
\left|\alpha_{k+1}\right| \leq \frac{c \exp \left(R^{\delta}\right)}{(R-1)^{k}\left|y_{k}(0)\right|}
$$


Let $R=1+k^{1 / \delta}$ and note that $R \leq(2 k)^{1 / \delta}$ for sufficiently large $k$, then

$$
\left|\alpha_{k+1}\right| \leq \frac{c \exp (2 k)}{k^{k / \delta}\left|y_{k}(0)\right|}
$$

and using the fact that $y_{k}(0) \rightarrow 1$ as $k \rightarrow \infty$ we compute

$$
\limsup _{k \rightarrow \infty} \frac{k \log k}{-\log \left|\alpha_{k}\right|} \leq \limsup _{k \rightarrow \infty} \frac{k \log k}{(k / \delta) \log k+\log \left|y_{k}(0)\right|-\log c-2 k}=\delta .
$$

This yields (4.2) since $\delta>\gamma$ was arbitrary.

\section{One-to-one correspondences for CMV matrices with super exponentially decaying Verblunsky coefficients}

In this section we present our second and third main results, which establish two one-to-one correspondences for CMV matrices with super exponentially decaying coefficients. More precisely, the first result we present shows that every entire function of finite growth order vanishing at the origin appears as the first component $u_{0}(z)$ of the Jost solution $\left\{u_{k}(z)\right\}_{k=0}^{\infty}$, of a unique CMV matrix $U$ with super exponentially decaying Verblunsky coefficients. Combined with Theorem 4.1 this provides a one-to-one correspondence between entire functions of finite growth order and super exponentially decaying Verblunsky coefficients. In addition, we note that our proof is constructive, and hence, it can be viewed as a solution to the inverse problem of finding a unique CMV matrix with super exponentially decaying Verblunsky coefficients and a prescribed first component $u_{0}(z)$ of its Jost solution $\left\{u_{k}(z)\right\}_{k=0}^{\infty}$.

Theorem 5.1. For every entire function $h(z)$ of finite growth order, there exists a unique CMV matrix $U$ with super exponentially decaying Verblunsky coefficients $\left\{\alpha_{k}\right\}_{k=1}^{\infty}$, that is,

$$
\limsup _{k \rightarrow \infty} \frac{k \log (k)}{-\log \left|\alpha_{k}\right|}<\infty
$$

such that the first component $u_{0}(z)$ of the Jost solution of $U$ on $\mathbb{C}$ satisfies $u_{0}(z)=z h(z)$.

Remark 5.2. (i) By Theorem 4.1 the limit in (5.1) equals the growth order of $h(z)$.

Proof. We start by showing the uniqueness part. Since the Schur functions are in one-to-one correspondence with CMV matrices by Geronimus' Theorem (cf. [26]), it suffices to show that $u_{0}(z)$ uniquely determines $f_{0}(z)$. Indeed, by the discussion in Section 3, we have the equivalent Jost solution $\left(\begin{array}{l}x(z) \\ y(z)\end{array}\right)$ of $(3.7)$ on $\mathbb{C}$ with $x_{0}(z)=u_{0}(z)$ and $y_{0}(z)$ being an entire function with no zeros on $\mathbb{D}$ by $(3.17)$ and $y_{0}(0)>0$ by (3.8). Thus, $y_{0}(z)$ on $\mathbb{D}$ is uniquely determined by $\left|y_{0}(z)\right|$ on $\partial \mathbb{D}$, and hence by the function $x_{0}(z)$ since $\left|y_{0}(z)\right|^{2}=1+\left|x_{0}(z)\right|^{2}$ on $\partial \mathbb{D}$ by (3.13). The Schur function $f_{0}(z)$ is then uniquely determined by $x_{0}(z)$ and $y_{0}(z)$ via $(3.16)$.

Next, we show the existence part by constructing the Verblunsky coefficients and the corresponding Jost solution $\left(\begin{array}{l}x(z) \\ y(z)\end{array}\right)$ of $(3.7)$ on $\mathbb{C}$. Let $x_{0}(z)=z h(z), z \in \mathbb{C}$, and define $y_{0}(z)$ on $\mathbb{D}$ by

$$
y_{0}(z)=\exp \left(\int_{-\pi}^{\pi} \frac{e^{i \theta}+z}{e^{i \theta}-z} \log \left(1+\left|x_{0}\left(e^{i \theta}\right)\right|^{2}\right) \frac{d \theta}{4 \pi}\right), \quad z \in \mathbb{D}
$$

and on $\mathbb{C} \backslash \overline{\mathbb{D}}$ by

$$
y_{0}(z)=\frac{1+x_{0}(z) x_{0}^{\#}(z)}{y_{0}^{\#}(z)}, \quad z \in \mathbb{C} \backslash \overline{\mathbb{D}}
$$


where $x_{0}^{\#}(z)$ and $y_{0}^{\#}(z)$ are given by (3.10). It follows from (5.2) that $y_{0}(z)$ has nontangential boundary values a.e. on $\partial \mathbb{D}$ and $\lim _{r \uparrow 1}\left|y_{0}\left(r e^{i \theta}\right)\right|^{2}=1+\left|x_{0}\left(e^{i \theta}\right)\right|^{2}$ for a.e. $\theta \in[0,2 \pi)$. By (5.3), a.e. on $\partial \mathbb{D}$ the boundary values of $y_{0}(z)$ from the outside of the unit circle match with the boundary values from the inside of the unit circle,

$$
\lim _{r \downarrow 1} y_{0}\left(r e^{i \theta}\right)=\frac{1+\left|x_{0}\left(e^{i \theta}\right)\right|^{2}}{\lim _{r \uparrow 1} \overline{y_{0}\left(r e^{i \theta}\right)}}=\frac{\lim _{r \uparrow 1}\left|y_{0}\left(r e^{i \theta}\right)\right|^{2}}{\lim _{r \uparrow 1} \overline{y_{0}\left(r e^{i \theta}\right)}}=\lim _{r \uparrow 1} y_{0}\left(r e^{i \theta}\right),
$$

and hence $y_{0}(z)$ is an entire function by Morera's theorem.

Now we recursively define the Verblunsky coefficients $\alpha_{k}$ and the entire functions $x_{k}(z), y_{k}(z), k \geq 1$. For $k=0,1,2, \ldots$, define $f_{k}(z)=z^{-1} x_{k}(z) / y_{k}(z), \alpha_{k+1}=-\overline{f_{k}(0)}$, and

$$
\left(\begin{array}{l}
x_{k+1}(z) \\
y_{k+1}(z)
\end{array}\right)=\frac{1}{\rho_{k+1}}\left(\begin{array}{cc}
\frac{1}{z} & \bar{\alpha}_{k+1} \\
\frac{1}{z} \alpha_{k+1} & 1
\end{array}\right)\left(\begin{array}{l}
x_{k}(z) \\
y_{k}(z)
\end{array}\right), \quad z \in \mathbb{C} .
$$

The definition of $x_{0}(z)$ and the above choice of the Verblunsky coefficients guarantees that $x_{k}(0)=0$ for all $k \geq 0$. Then, since $y_{0}(0)$ is finite by (3.8), it follows that the functions $x_{k+1}(z)$ and $y_{k+1}(z)$ defined in (5.5) have no poles at the origin and hence are entire for all $k \geq 0$. Moreover, (5.5) is equivalent to (3.7), hence the constructed sequence $\left(\begin{array}{l}x(z) \\ y(z)\end{array}\right)=\left\{\left(\begin{array}{l}x_{k}(z) \\ y_{k}(z)\end{array}\right)\right\}_{k=0}^{\infty}$ is a solution of (3.7) on $\mathbb{C}$. Using (3.7) at $z=0$ we get $y_{k}(0)=y_{0}(0) \prod_{n=1}^{k} \rho_{n} \geq 0$. Thus, $y_{k}(0)$ is monotonically decreasing as $k \rightarrow \infty$ and as a result $y_{k}(0)$ has a limit $Y=\lim _{k \rightarrow \infty} y_{k}(0) \geq 0$.

Finally, recalling the estimates (4.14) and (4.17) obtained in the proof of the previous theorem, we get for any $R>1$ that $\left\|x_{k}\right\| \rightarrow 0$ and $\left\|y_{k}-y_{k}(0)\right\| \rightarrow 0$ as $k \rightarrow \infty$. Hence, for every $z \in \mathbb{C}$ we have $x_{k}(z) \rightarrow 0$ and $y_{k}(z)-y_{k}(0) \rightarrow 0$ as $k \rightarrow \infty$. The latter one implies that $y_{k}(z) \rightarrow Y$ for all $z \in \mathbb{C}$ as $k \rightarrow \infty$ since $y_{k}(0)$ has the limit $Y$. Taking $k \rightarrow \infty$ in (3.13) yields $|Y|^{2}=1$, and hence, $y_{k}(z) \rightarrow 1$ for all $z \in \mathbb{C}$ as $k \rightarrow \infty$. Thus, the constructed solution $\left(\begin{array}{l}x(z) \\ y(z)\end{array}\right)$ is the Jost solution of (3.7) on $\mathbb{C}$ corresponding to the constructed sequence of Verblunsky coefficients. The super exponential decay condition (5.1) follows from Theorem 4.1 as $u_{0}(z)=x_{0}(z)=z h(z)$ is of finite growth order.

In our second result we continue the study of CMV matrices with super exponentially decaying coefficients. For the Jost solutions $\left\{u_{k}(z)\right\}_{k=0}^{\infty}$ associated with such matrices we describe the distribution of zeros of $u_{0}(z)$. More precisely, we show that the reciprocals of the nonzero roots of $u_{0}(z)$ are in $\ell^{p}$ for all $p>\delta>0$ whenever $\left|\alpha_{k}\right| \leq c k^{-k / \delta}$. This describes the distribution of roots of $u_{0}(z)$ near infinity. For the roots of $u_{0}(z)$ on the unit disk we establish the lower bound $\left|\alpha_{1}\right|<\prod_{\left|z_{k}\right|<1}\left|z_{k}\right|$, where $\alpha_{1}$ is the first Verblunsky coefficient. There is a long history of similar results for Jacobi and Schrödinger operators in which zeros of the corresponding Jost functions (called resonances) were studied under various assumptions on the background operators and their compact perturbations, see for instance $[7,8,11,14,15,18,23,32]$ and the references therein.

There is also a related inverse problem of constructing/recovering a CMV matrix from a prescribed set of zeros of $u_{0}(z)$. It is easy to see that this problem does not have a unique solution in general. Indeed, the functions $u_{0}(z)=z e^{z}$ and $u_{0}(z)=b z, b \in \mathbb{C}$, correspond to different CMV matrices by Theorem 5.1 yet all have the only zero at the origin. It turns out that these examples illustrate essentially all possible cases of nonuniqueness. In our result below we impose an additional assumption of fast super exponential decay $\left|\alpha_{k}\right| \leq c k^{-k / \delta}, 0<\delta<1$ which, by Theorem 4.1, rules out presence of an exponential factor and assume that $\alpha_{1}$ is known which fixes the multiplicative constant $b$. More precisely, we show that under the assumption of fast super exponential decay of Verblunsky coefficients the zeros of $u_{0}(z)$ together with the value of the first Verblunsky coefficient $\alpha_{1}$ uniquely determine a CMV matrix. In fact, we show that there is a unique CMV matrix for every prescribed set of zeros for $u_{0}(z)$ and every prescribed value for the first Verblunsky coefficient $\alpha_{1}$ as long as they satisfy the restrictions of the distribution of zeros result mentioned above. Thus, for each fixed value of $\alpha_{1}$ we obtain a one-to-one correspondence between the class of CMV matrices with $\left|\alpha_{k}\right| \leq c k^{-k / \delta}, 0<\delta<1$, and the collection of sets of zeros for $u_{0}(z)$ allowed by the distribution of zeros result. 
In the context of Jacobi and Schödinger operators the inverse problem of recovering an operator from the set of zeros of its Jost function is known as the inverse resonance problem, see for instance $[1-3,12,13,16,17,19-21,33]$ and the references therein. Our result is a CMV analog of these inverse resonance problems. The uniqueness part of our result has appeared earlier in [31] under stronger decay assumptions on Verblunsky coefficients. Also uniqueness and stability of the inverse resonance problem associated with the Jost function $\psi_{0}(z)$ of a CMV matrix has been treated in [22].

Theorem 5.3. Let $\left\{z_{k}\right\}_{k=1}^{K} \subset \mathbb{C} \backslash\{0\}, K \in\{0,1, \ldots, \infty\}$, be a sequence satisfying

$$
\inf \left\{\delta \geq 0: \sum_{k=1}^{K} \frac{1}{\left|z_{k}\right|^{\delta}}<\infty\right\} \leq \gamma<1
$$

and let $a \in \mathbb{D}$ be such that

$$
0<|a|<\prod_{\left|z_{k}\right|<1}\left|z_{k}\right|
$$

Then in the class of CMV matrices with Verblunsky coefficients satisfying

$$
\limsup _{k \rightarrow \infty} \frac{k \log (k)}{-\log \left|\alpha_{k}\right|} \leq \gamma<1
$$

there exists a unique $C M V$ matrix $U$ with the Jost solution $\left\{u_{k}(z)\right\}_{k=0}^{\infty}$ on $\mathbb{C}$ such that $\alpha_{1}=a$ and the roots of $u_{0}(z)$ repeated according to their multiplicities are precisely $\{0\} \cup\left\{z_{k}\right\}_{k=1}^{K}$.

Conversely, let $U$ be a CMV matrix with Verblunsky coefficients $\left\{\alpha_{k}\right\}_{k=1}^{\infty}$ satisfying $\alpha_{1} \neq 0$ and (5.8) and let $\left\{u_{k}(z)\right\}_{k=0}^{\infty}$ be the Jost solution of $U$ on $\mathbb{C}$. Then the nonzero roots of $u_{0}(z)$ repeated according to their multiplicities $\left\{z_{k}\right\}_{k=1}^{K}$ satisfy (5.6) and $a=\alpha_{1}$ satisfies (5.7).

Remark 5.4. (i) The value of the infimum in (5.6) is the exponent of convergence for $\left\{z_{k}\right\}_{k=1}^{K}$.

(ii) Due to the equivalence, the nonstrict inequalities in (5.6) and (5.8) can be replaced by the equalities.

(iii) It follows from the proof that the restriction $\gamma<1$ in (5.6) is only necessary for the uniqueness part of the forward direction. The existence part and the converse direction continue to hold with the restriction $\gamma<1$ dropped from (5.6) and (5.8).

(iv) The theorem also extends to the case where $u_{0}(z)$ has a zero of order $k_{0} \geq 1$ at the origin. In this case the Verblunsky coefficients satisfy $\alpha_{1}=\cdots=\alpha_{k_{0}-1}=0, \alpha_{k_{0}}=a \neq 0$.

Proof. We start by showing the uniqueness part of the forward direction. As discussed in Section 3 (cf. (3.5), (3.6)), the Jost solution $\left\{u_{k}(z)\right\}_{k=0}^{\infty}$ of a CMV matrix $U$ on $\mathbb{C}$ is equivalent to the Jost solution $\left(\begin{array}{l}x(z) \\ y(z)\end{array}\right)$ of $(3.7)$ on $\mathbb{C}$ with $u_{0}(z)=x_{0}(z)$. By Theorem 4.1, the function $x_{0}(z)$ is entire of growth order strictly less than 1 for any CMV matrix $U$ with Verblunsky coefficients satisfying (5.8). Thus, by the Hadamard factorization theorem,

$$
x_{0}(z)=b z \Pi(z), \quad z \in \mathbb{C},
$$

where $b \in \mathbb{C} \backslash\{0\}$ and

$$
\Pi(z)=\prod_{k=1}^{K}\left(1-\frac{z}{z_{k}}\right), \quad z \in \mathbb{C} .
$$

The function $y_{0}(z)$ is also an entire function and, by $(3.17), y_{0}(z)$ has no zeros on $\mathbb{D}$. Hence $y_{0}(z)$ is an outer function on $\mathbb{D}$. Then since $y_{0}(0)>0$ by $(3.8)$ and $\left|y_{0}(z)\right|^{2}=1+\left|x_{0}(z)\right|^{2}=1+|b|^{2}|\Pi(z)|^{2}$ on $\partial \mathbb{D}$ by (3.13) and (5.9), we have

$$
y_{0}(z)=\exp \left(\int_{-\pi}^{\pi} \frac{e^{i \theta}+z}{e^{i \theta}-z} \log \left(1+|b|^{2}\left|\Pi\left(e^{i \theta}\right)\right|^{2}\right) \frac{d \theta}{4 \pi}\right), \quad z \in \mathbb{D} .
$$


Since $\alpha_{1}=a$, it follows from (2.9), (3.16), (5.9), and (5.11) that

$$
\begin{aligned}
& \arg (-\bar{a})=\arg (b) \\
& \log |a|=\int_{-\pi}^{\pi} \log \left(\frac{|b|^{2}}{1+|b|^{2}\left|\Pi\left(e^{i \theta}\right)\right|^{2}}\right) \frac{d \theta}{4 \pi} .
\end{aligned}
$$

It is easy to see that the RHS of (5.13) is monotone increasing w.r.t. $|b|$, hence the value of $b$ is uniquely determined by $a$ via (5.12) and (5.13). Thus, the first component of the Jost solution $u_{0}(z)=x_{0}(z)$ is uniquely determined by $a$ and $\left\{z_{k}\right\}_{k=1}^{K}$, and hence, the CMV matrix $U$ is unique by Theorem 5.1.

Next, we show the existence part of the forward direction. By Theorem 5.1 it suffices to construct an appropriate function $u_{0}(z)$. Define the function $\Pi(z)$ according to $(5.10)$ and let $u_{0}(z)=b z \Pi(z)$ with some constant $b \in \mathbb{C} \backslash\{0\}$ to be determined later. Then it follows that $\Pi(z)$ and hence also $u_{0}(z)$ are entire functions of growth order at most $\gamma$. Indeed, for any $\delta \in(0,1)$ there exist a constant $c=c(\delta)>0$ such that $\log |1-z| \leq \log (1+|z|) \leq c|z|^{\delta}, z \in \mathbb{C}$. Then utilizing (5.6) with $\delta \in(\gamma, 1)$ gives

$$
\limsup _{R \rightarrow \infty} \frac{\log \log \max _{|z|=R}|\Pi(z)|}{\log R} \leq \limsup _{R \rightarrow \infty} \frac{\log \left(\sum_{k=1}^{K} c \frac{R^{\delta}}{\left|z_{k}\right|^{\delta}}\right)}{\log R}=\delta .
$$

Since $\delta \in(\gamma, 1)$ was arbitrary, the growth order claim follows.

We determine the constant $b$ from the requirement $\alpha_{1}=a$ which is equivalent to (5.12) and (5.13) as was discussed in the previous part. The equation (5.12) is trivially solvable, thus, it remains to verify that (5.13) has a solution. It suffices to show that $\log |a|$ is in the range of the RHS of (5.13) as $|b|$ runs through $(0, \infty)$. Since the RHS of $(5.13)$ is monotone increasing and continuous w.r.t. $|b|$, the range of the RHS of (5.13) is an interval. The end points of this interval are the limits of the RHS of (5.13) as $|b| \rightarrow 0$ and $|b| \rightarrow \infty$ which, by the monotone convergence theorem, are equal to $-\infty$ and $\int_{-\pi}^{\pi}-\log |\Pi(z)| \frac{d \theta}{2 \pi}$, respectively. By Jensen's formula, we get the following expression for the right end point of the interval,

$$
-\int_{-\pi}^{\pi} \log |\Pi(z)| \frac{d \theta}{2 \pi}=-\log |\Pi(0)|-\sum_{\left|z_{k}\right|<1} \log \frac{1}{\left|z_{k}\right|}=\sum_{\left|z_{k}\right|<1} \log \left|z_{k}\right| .
$$

Thus, it follows from (5.7) that $\log |a|$ is in the rage of the RHS of (5.13), and hence, (5.13) has a solution.

Finally, we show the converse direction. It follows from the Theorem 5.1 that the function $u_{0}(z)$ is entire of growth order at most $\gamma<1$. Then by [10, Thm.7.8.2] the zeros of $u_{0}(z)$ have the exponent of convergence at most $\gamma$, that is, (5.6) holds. Moreover, applying Jensen's formula to the Schur function $f_{0}(z)$ and recalling that $f_{0}(0)=-\bar{\alpha}_{1}$ by $(2.9)$ yield

$$
\log \left|\alpha_{1}\right|=\frac{1}{2 \pi} \int_{-\pi}^{\pi} \log \left|f\left(e^{i \theta}\right)\right| d \theta-\sum_{\left|z_{k}\right|<1} \log \frac{1}{\left|z_{k}\right|} \leq \sum_{\left|z_{k}\right|<1} \log \left|z_{k}\right|,
$$

that is, (5.7) holds for $a=\alpha_{1}$.

\section{References}

[1] B. M. Brown, I. Knowles, R. Weikard. On the inverse resonance problem. J. London Math. Soc., 68 (2003), no. 2, 383-401.

[2] B. M. Brown, S. Naboko, R. Weikard. The inverse resonance problem for Jacobi operators. Bull. London Math. Soc., 37 (2005), no. 5, 727-737.

[3] B. M. Brown, S. Naboko, R. Weikard. The inverse resonance problem for Hermite operators. Constr. Approx., 30 (2009), no. 2, 155-174.

[4] A. Bunse-Gerstner, L. Elsner. Schur parameter pencils for the solution of the unitary eigenproblem. Linear Algebra Appl., 154/156 (1991), 741-778.

[5] M. J. Cantero, L. Moral, L. Velázquez. Five-diagonal matrices and zeros of orthogonal polynomials on the unit circle. Linear Algebra Appl., 362 (2003), 29-56. 
[6] D. Damanik, B. Simon. Jost functions and Jost solutions for Jacobi matrices. II. Decay and analyticity. Int. Math. Res. Not. (2006), Art. ID 19396, 1-32.

[7] L. D. Faddeyev. The inverse problem in the quantum theory of scattering. J. Mathematical Phys., 4 (1963), $72-104$.

[8] R. Froese. Asymptotic distribution of resonances in one dimension. J. Differential Equations, 137 (1997), no. 2, 251272.

[9] F. Gesztesy, M. Zinchenko. Weyl-Titchmarsh theory for CMV operators associated with orthogonal polynomials on the unit circle. J. Approx. Theory, 139 (2006), no. 1-2, 172-213.

[10] L.-S. Hahn, B. Epstein. Classical complex analysis. Jones \& Bartlett Learning, 1996.

[11] M. Hitrik. Bounds on scattering poles in one dimension. Comm. Math. Phys., 208 (1999), no. 2, $381-411$.

[12] A. Iantchenko, E. Korotyaev. Periodic Jacobi operator with finitely supported perturbation on the half-lattice. Inverse Problems, 27 (2011), no. 11, 115003, 26.

[13] A. Iantchenko, E. Korotyaev. Periodic Jacobi operator with finitely supported perturbations: the inverse resonance problem. J. Differential Equations, 252 (2012), no. 3, 2823-2844.

[14] A. Iantchenko, E. Korotyaev. Resonances for periodic Jacobi operators with finitely supported perturbations. J. Math. Anal. Appl., 388 (2012), no. 2, 1239-1253.

[15] E. Korotyaev. Inverse resonance scattering on the half line. Asymptot. Anal., 37 (2004), no. 3-4, $215-226$.

[16] E. Korotyaev. Stability for inverse resonance problem. Int. Math. Res. Not. (2004), no. 73, 3927-3936.

[17] E. Korotyaev. Inverse resonance scattering for Jacobi operators. Russ. J. Math. Phys., 18 (2011), no. 4, $427-439$.

[18] E. Korotyaev. Resonance theory for perturbed Hill operator. Asymptot. Anal., 74 (2011), no. 3-4, $199-227$.

[19] M. Marletta, S. Naboko, R. Shterenberg, R. Weikard. On the inverse resonance problem for Jacobi operators - uniqueness and stability. J. Anal. Math., 117 (2012), 221-247.

[20] M. Marletta, R. Shterenberg, R. Weikard. On the inverse resonance problem for Schrödinger operators. Comm. Math. Phys., 295 (2010), no. 2, 465-484.

[21] M. Marletta, R. Weikard. Stability for the inverse resonance problem for a Jacobi operator with complex potential. Inverse Problems, 23 (2007), no. 4, 1677-1688.

[22] R. Shterenberg, R. Weikard, M. Zinchenko. Stability for the inverse resonance problem for the CMV operator. Proc. Sympos. Pure Math., 87 (2013), 315-326.

[23] B. Simon. Resonances in one dimension and Fredholm determinants. J. Funct. Anal., 178 (2000), no. 2, $396-420$.

[24] B. Simon. Orthogonal polynomials on the unit circle: new results. Int. Math. Res. Not. (2004), no. 53, $2837-2880$.

[25] B. Simon. OPUC on one foot. Bull. Amer. Math. Soc. (N.S.), 42 (2005), no. 4, 431-460 (electronic).

[26] B. Simon. Orthogonal polynomials on the unit circle. Part 1: Classical theory, American Mathematical Society Colloquium Publications, vol. 54, American Mathematical Society, Providence, RI, 2005.

[27] B. Simon. Orthogonal polynomials on the unit circle. Part 2: Spectral theory, American Mathematical Society Colloquium Publications, vol. 54, American Mathematical Society, Providence, RI, 2005.

[28] B. Simon. CMV matrices: five years after. J. Comput. Appl. Math., 208 (2007), no. 1, 120-154.

[29] G. Teschl. Jacobi operators and completely integrable nonlinear lattices. Mathematical Surveys and Monographs, vol. 72, American Mathematical Society, Providence, RI, 2000.

[30] D. S. Watkins. Some perspectives on the eigenvalue problem. SIAM Rev., 35 (1993), no. 3, 430-471.

[31] R. Weikard, M. Zinchenko. The inverse resonance problem for CMV operators. Inverse Problems, 26 (2010), no. 5, $55012-55021$.

[32] M. Zworski. Distribution of poles for scattering on the real line. J. Funct. Anal., 7 (1987), no. 2, $277-296$.

[33] M. Zworski. A remark on isopolar potentials. SIAM J. Math. Anal., 32 (2001), no. 6, 1324-1326 (electronic). 